\title{
Factores que determinan el éxito o fracaso de proyectos forestales comunitarios con financiamiento gubernamental en México
}

\author{
Determinants of success or failure on community forestry projects with government \\ funding in Mexico
}

\author{
Humberto Martínez-Bautista a , Francisco José Zamudio Sánchez ${ }^{\text {a*}}$, Arturo A Alvarado-Segura ${ }^{\text {a,b }}$, \\ Hugo Ramírez Maldonado a, Mario Fuentes Salinas ${ }^{a}$ \\ *Autor de correspondencia: ${ }^{a}$ Universidad Autónoma Chapingo, División de Ciencias Forestales, \\ Km 38.5 Carretera México-Texcoco, C.P. 56230, Texcoco, Estado de México, tel.: (52) 59595470 78, zafra1949@gmail.com \\ ${ }^{\mathrm{b}}$ Instituto Tecnológico Superior del Sur del Estado de Yucatán, C.P. 97880, Oxkutzcab, Yucatán, México.
}

\section{SUMMARY}

The purpose of this research was to determine the social, economic and environmental factors that influence the probability of success (failure) of timber-yielding projects under government funding in Mexico. Results are relevant for designing effective public programs focused on diminishing biodiversity loss and poverty persistence in the region. Following the scheme of community forestry in Latin America, between 2004 and 2008, the World Bank funded 96 projects in 68 Mexican communities. We focused on nine factors and their levels (explanatory variables) to explain the current status of timber-yielding projects (response variable). Our main information sources were official reports and structured interviews to people who know the community forestry program in Mexico (key informants) and chosen in a non-random way. Using association analyses, correlation, and logistic regression models, we found that social capital was the most important factor to explain both the probability of success and the one of failure. A high social capital level explained more than $43 \%$ of success probability while low and medium social capital levels explained almost $55 \%$ of failure probability. Furthermore, we found that the type of project is a strong determinant of success or failure; continuation of a project is not necessarily related to probability of success; and the type of social property does not determine the project success or failure. Our results agree with the theory of community forestry development.

Key words: community forestry development, community forestry, social capital, drivers of success or failure.

\section{RESUMEN}

El propósito fue determinar los factores sociales, económicos y ambientales que influencian la probabilidad de éxito (o fracaso) de proyectos maderables con financiamiento gubernamental en México. Los resultados tienen relevancia para diseñar programas públicos efectivos orientados a mitigar la pérdida de la biodiversidad y la persistencia de la pobreza en la región. Siguiendo el esquema de la silvicultura comunitaria en América Latina, el Banco Mundial financió en México, entre 2004 y 2008 , 96 proyectos en 68 comunidades. Fueron utilizados nueve factores y sus niveles (variables explicativas) para explicar el éxito o fracaso de los proyectos (variable respuesta). Las fuentes principales de información fueron reportes oficiales y entrevistas estructuradas a conocedores de los programas de silvicultura comunitaria (informantes clave) elegidos de forma dirigida. Usando análisis de asociación, correlación y modelos de regresión logística se encontró que el capital social fue el factor más importante para explicar tanto la probabilidad de éxito como de fracaso. Un nivel de capital social alto explicó más del $43 \%$ de la probabilidad de éxito mientras que los niveles bajo y medio explicaron casi el $55 \%$ de la probabilidad de fracaso. También se encontró que: el tipo de proyecto influyó fuertemente en el éxito o fracaso del mismo; la continuación de los proyectos, no necesariamente incrementó su probabilidad de éxito; por último, el tipo de propiedad social no determinó el éxito o fracaso de los proyectos. Los resultados están en concordancia con la teoría del desarrollo forestal comunitario.

Palabras clave: desarrollo forestal comunitario, silvicultura comunitaria, capital social, factores de éxito o fracaso.

\section{INTRODUCCIÓN}

La silvicultura comunitaria ha sido promovida en países de América Latina, entre los que destacan: México, Costa Rica, Honduras, Perú, Bolivia y Brasil (Pokorny et al. 2009). Esta perspectiva plantea que la silvicultura ad- quiere sentido solo cuando satisface las necesidades de las personas que habitan el bosque. Sus principales funciones son: proveer combustible y otros bienes primarios, proveer alimento y estabilidad ambiental, y generar empleo e ingreso en las comunidades rurales (FAO 1978, Leslie 1987). Sin embargo, la sostenibilidad de la silvicultura co- 
munitaria es un reto debido a la falta de continuidad del financiamiento y del potencial limitado para extender el éxito cuando ocurre (Pokorny et al. 2009). En un escenario donde la persistencia de la pobreza está asociada con la destrucción del bosque, la silvicultura comunitaria se perfila como una opción para proteger la biodiversidad así como disminuir la pobreza de las zonas rurales (Luján 2003, Pokorny et al. 2009, Alcorn 2014). América Latina posee un liderazgo en el desarrollo de proyectos de silvicultura comunitaria y desde el punto de vista de la biodiversidad, incluye cinco de las diez áreas con mayor diversidad en el mundo: México, Brasil, Perú, Ecuador y Colombia (Alcorn 2014).

En México existen núcleos agrarios (ejidos y comunidades), poseedores de tierras de propiedad colectiva, que juegan un papel crucial sobre los bosques y selvas. En América Latina se estima que el $32 \%$ de bosques y selvas pertenecen a comunidades rurales, porcentaje que en México alcanza el $60 \%$ (Bray et al. 2003, Madrid et al. 2009, Pokorny et al. 2009). Por lo que es relevante el entendimiento de los factores que determinan el éxito o el fracaso de los proyectos forestales comunitarios, para identificar las condiciones de éxito y disminuir los casos de fracaso, en el diseño de políticas públicas efectivas. Además, las experiencias de éxito o de fracaso en la silvicultura comunitaria pueden servir como directrices para instrumentar los proyectos de Reducción de Emisiones causadas por la Deforestación y la Degradación de los bosques (Pokorny et al. 2009, Alcorn 2014).

La probabilidad de éxito o fracaso de los proyectos forestales con financiamiento gubernamental en México, está condicionada por factores sociales, económicos y ambientales asociados con los núcleos agrarios (Bray y Merino 2004, López et al. 2010). Los proyectos con éxito dentro de la silvicultura comunitaria surgen de un conjunto de factores como: el capital social, el capital natural (Bray y Merino 2004), el capital humano (CONAFOR 2003), y factores externos como las condiciones de mercado, el acceso a créditos, y la intervención de programas de política pública (Madrid et al. 2009). El análisis de experiencias exitosas sugiere que el fortalecimiento tanto del capital social como del capital humano, así como de las instituciones comunitarias, son factores relevantes para el desarrollo comunitario, la conservación y el manejo sostenible de los recursos (Merino 2003, Bray et al. 2007, Madrid et al. 2009, López et al. 2010). Un nivel de capital social alto es una condición necesaria, pero no suficiente, para el éxito; además, amortigua los efectos negativos de otros factores adversos (Agrawal 1995) y es imprescindible para el desarrollo y preservación de otros tipos de activos como los capitales natural, físico y humano (Rubio et al. 2007).

En esta investigación se entiende por empresa forestal comunitaria a la estructura organizativa y administrativa que cuenta con sistemas contables y de rendición de cuentas, encargada de los proyectos comunitarios para el aprovechamiento forestal (Gerez y Purata 2008), cuyos dirigentes son designados por las asambleas. Este instrumento es fundamental para consolidar la formación de capital social e impulsar el aprovechamiento forestal sostenible (CONAFOR 2012). En México el número de núcleos agrarios con permiso de aprovechamiento forestal (empresas forestales comunitarias) entre 1992 y 2002, fue de aproximadamente 2.300 , lo que representó el $15 \%$ del universo de núcleos agrarios (Bray et al. 2007).

La instrumentación de empresas forestales comunitarias reduce la pobreza y la marginación, debido a que: a) genera empleos, b) diversifica las actividades económicas, c) genera beneficios sociales, d) organiza el aprovechamiento forestal, e) propicia economías de escala, y f) da valor agregado a los productos forestales. Así, la operación de dichas empresas ofrece una oportunidad de reducir la pobreza, prevenir la migración, generar capital humano, revitalizar el campo y proteger los recursos naturales (Bray et al. 2003, Madrid et al. 2009). Para impulsar esta estrategia, de 2004 a 2008 se desarrollaron en México 198 proyectos (maderables y no maderables) con financiamiento del Banco Mundial en seis estados que concentran más del $47 \%$ de los ejidos y comunidades forestales: Durango, Guerrero, Jalisco, Michoacán, Oaxaca y Quintana Roo (CONAFOR 2008, INEGI 2008).

En la evaluación de los proyectos maderables financiados de 2004 a 2008, se reportaron indicadores financieros de éxito: un valor presente neto de 63,8 millones de pesos, una tasa interna de retorno de $20,16 \%$, una relación beneficio-costo de 1,78 y un periodo de recuperación de seis años (CONAFOR 2008). Sin embargo, debido a las escasas fuentes de información, aún no se han investigado los factores subyacentes que determinan el éxito o fracaso de este tipo de proyectos. Por lo que en esta investigación el objetivo fue identificar los factores sociales, económicos y ambientales que determinan la probabilidad del éxito o fracaso de los proyectos forestales comunitarios con financiamiento gubernamental en México. Un proyecto se considera exitoso cuando funciona, es sostenible y genera empleos; en caso contrario, se considera un fracaso. Los proyectos forestales comunitarios están vinculados con la capacidad de los núcleos agrarios de trabajar juntos, establecer acuerdos de participación y construir redes de cooperación, esto es, del nivel de capital social. Por lo tanto, una hipótesis que debe ponerse a prueba es que el nivel del capital social condiciona el éxito o el fracaso de los proyectos forestales comunitarios.

\section{MÉTODOS}

Materiales. Se utilizó información proveniente de 96 proyectos maderables con financiamiento gubernamental, ejercidos por 68 núcleos agrarios (apéndice 1). Se aplicó un cuestionario estructurado de 11 preguntas a un grupo de informantes clave, conformado por 12 expertos seleccionados de forma dirigida, por su conocimiento de los núcleos agrarios intervenidos y la situación de los proyectos. 
Por cada proyecto maderable se investigó lo relacionado con el capital social, funcionamiento de la empresa forestal comunitaria, programa de manejo forestal y asistencia técnica de los núcleos agrarios.

Para indagar la consistencia de la información proporcionada por los informantes clave fueron realizadas dos acciones: 1) En el estado de Quintana Roo, se aplicaron tres entrevistas directamente a las autoridades agrarias. En los ejidos de San Francisco Botes y Noh-Bec se obtuvo una calificación mayor del capital social que la reportada por el informante clave; en cambio, en el ejido Tres Garantías, ambas calificaciones coincidieron. Para el análisis de datos de Quintana Roo, en los tres casos se empleó la información directa de las autoridades. 2) En el estado de Oaxaca, la información proporcionada por el informante clave de los núcleos agrarios La Trinidad, Santa Catarina Ixtepeji e Ixtlán de Juárez, se confrontó con el estudio realizado por Rubio et al. (2007), sin encontrar evidencia de sesgos.

Con base en las reglas para el financiamiento de los proyectos de desarrollo forestal comunitario es notable que los informantes clave no tuvieran algún interés personal, que indujera posibles sesgos en sus respuestas. Además ninguno tenía relación con el programa al momento de la entrevista, excepto el informante de Oaxaca, en cuyo caso la información fue validada con tres proyectos, los cuales pueden considerarse una muestra aleatoria.
Factores de estudio. Con base en la revisión documental se seleccionó un conjunto de factores explicativos (cuadro 1) que están relacionados con la situación actual de los proyectos (operando, ampliado, suspendido temporalmente o abandonado).

Descripción de los factores. a) Capital social, es el conjunto de relaciones de confianza y normas de reciprocidad, redes de intercambio y formas de participación civil, y reglas o instituciones tanto formales como informales (Ostrom y Ahn 2003). b) Indigenismo, se refiere a núcleos agrarios con población que habla algún dialecto nativo. c) Tipo de propiedad, se refiere a la forma de propiedad social que corresponde a ejidos y comunidades en México (Bray y Merino 2004). d) Finalidad del proyecto, es el giro o actividad que realizan con el proyecto maderable. e) Tipología del productor, es la clasificación de los núcleos agrarios productores en cuatro categorías de acuerdo a su grado de aprovechamiento (Bray y Merino 2004): productores potenciales (tipo I), productores que venden su madera en pie (tipo II), productores de materias primas forestales (tipo III) y productores con capacidad de transformación y comercialización (tipo IV). f) Funcionamiento de la empresa, se refiere a la operación de la empresa forestal del núcleo agrario. g) Grado de avance del proyecto, es el tiempo de operación del proyecto. h) Superficie forestal, se refiere al recurso forestal con que cuenta el ejido o comunidad.

Cuadro 1. Factores de investigación, niveles y fuentes de información.

Research factors, levels and sources of information.

\begin{tabular}{|c|c|c|c|}
\hline Bloque & Factores & Niveles & Fuentes de información \\
\hline Variable respuesta & Estatus del proyecto & $\begin{array}{l}\text { Éxito } \\
\text { Fracaso }\end{array}$ & $\begin{array}{l}\text { Informantes clave y para Quintana Roo una } \\
\text { autoridad agraria. }\end{array}$ \\
\hline \multirow[t]{3}{*}{ Social } & Capital social & $\begin{array}{l}\text { Alto, Medio y } \\
\text { Bajo }\end{array}$ & $\begin{array}{l}\text { Informantes clave y para Quintana Roo una } \\
\text { autoridad agraria. }\end{array}$ \\
\hline & Indigenismo & $\begin{array}{l}\text { Indígena } \\
\text { No indígena }\end{array}$ & Comisión Nacional Forestal. \\
\hline & Tipo de propiedad & $\begin{array}{l}\text { Comunidad } \\
\text { Ejido }\end{array}$ & $\begin{array}{l}\text { Padrón e Historial de Núcleos Agrarios } \\
\text { (PHINA) } \\
\text { http://phina.ran.gob.mx/phina2/ }\end{array}$ \\
\hline \multirow[t]{4}{*}{ Económico } & Finalidad del proyecto & $\begin{array}{l}\text { Adquisición, Equipamiento, } \\
\text { Aprovechamiento, Abastecimiento, } \\
\text { Estufas de secado y Muebles }\end{array}$ & $\begin{array}{l}\text { Clasificación propia con base en el concepto } \\
\text { de apoyo otorgado. }\end{array}$ \\
\hline & Tipología del productor & I, II, III y IV & Comisión Nacional Forestal. \\
\hline & $\begin{array}{l}\text { Funcionamiento de la } \\
\text { empresa }\end{array}$ & $\begin{array}{l}\text { Sí empresa } \\
\text { No empresa }\end{array}$ & $\begin{array}{l}\text { Informantes clave y para Quintana Roo una } \\
\text { autoridad agraria. }\end{array}$ \\
\hline & $\begin{array}{l}\text { Grado de avance del } \\
\text { proyecto }\end{array}$ & $\begin{array}{l}\text { Continuación } \\
\text { Nuevo }\end{array}$ & $\begin{array}{l}\text { Informantes clave y para Quintana Roo una } \\
\text { autoridad agraria. }\end{array}$ \\
\hline Ambiental & $\begin{array}{l}\text { Superficie forestal / } \\
\text { Superficie total }\end{array}$ & $\begin{array}{l}\text { Superficie forestal alta }(\geq 0,5) \\
\text { Superficie forestal baja }(<0,5)\end{array}$ & $\begin{array}{l}\text { Comisión Nacional Forestal y Padrón e } \\
\text { Historial de Núcleos Agrarios (PHINA) } \\
\text { http://phina.ran.gob.mx/phina2/ }\end{array}$ \\
\hline
\end{tabular}


i) Superficie total, es la dotación de tierras entregada al núcleo agrario por el Gobierno Mexicano.

Codificación de los niveles de los factores. Se utilizó la función indicadora para transformar cada nivel de los factores a binario. Por ejemplo, el capital social tiene tres niveles: alto, medio y bajo; en el nivel alto, se asignó el valor de 1 si pertenece a él y 0 en caso contrario. Se usó el mismo procedimiento para los otros niveles.

Determinación de la asociación. Se utilizó la prueba jicuadrada de Pearson para analizar la asociación entre el éxito o fracaso de un proyecto forestal maderable con cada uno de los factores. Esta prueba no paramétrica se basa en la distribución probabilística ji-cuadrada y sirve para medir la compatibilidad existente entre el valor del estadístico ji-cuadrada y la hipótesis de independencia.

Determinación de la correlación. Se utilizó la matriz de correlaciones tetracóricas para evaluar la correlación entre el éxito o fracaso de un proyecto forestal, con cada uno de los niveles de los factores; y al mismo tiempo explorar problemas de colinealidad entre factores. El coeficiente de correlación tetracórica es una prueba paramétrica que estima la relación entre dos variables dicotómicas asumiendo una distribución normal bivariada. Esta prueba es la mejor alternativa en presencia de datos binarios y tiene la misma interpretación que el coeficiente de correlación de Pearson (Ledesma et al. 2011).

Análisis estadístico. Se utilizó la regresión logística para evaluar la aportación de los factores y su significancia estadística. En la construcción del modelo se consideraron los niveles de los factores que se encontraron relacionados con el éxito o con el fracaso de los proyectos maderables. Es decir, si la correlación fue positiva se incluyó en el modelo de éxito, en caso contrario, en el de fracaso. La regresión logística se usa para describir la relación existente entre una variable respuesta dicotómica y una o más variables explicativas que pueden ser tanto categóricas como continuas. Los análisis estadísticos se realizaron con el paquete Data Analysis and Statistical Software (STATA) versión 12.

\section{RESULTADOS}

Descripción de la población de estudio. Todos los núcleos agrarios contaban con programa de manejo forestal maderable y servicios técnicos al momento de desarrollar los proyectos. En lo que se refiere al tipo de propiedad, $50 \%$ fueron comunidades y $50 \%$ fueron ejidos. La misma distribución presentó el factor indigenismo, 50 \% indígenas y $50 \%$ no indígenas. De acuerdo con la finalidad del proyecto maderable se encontraron 23 proyectos para adquisición de aserraderos, 21 para equipamiento de aserraderos, 19 para abastecimiento forestal, 12 para aprovechamiento fo- restal, 13 para instalación de estufas de secado y ocho para fabricación de muebles.

Los proyectos maderables fueron desarrollados en su mayoría por productores tipo IV con 72 proyectos, seguidos por los productores tipo III con 19 proyectos y, por último, sólo cinco proyectos fueron realizados por productores tipo II. No se encontraron proyectos maderables para los productores tipo I, lo cual indicó una congruencia del programa en el financiamiento de dichos proyectos.

Se encontró un total de 74 proyectos funcionando y generando empleos, a los cuales se les catalogó como éxitos; los restantes 22 proyectos fueron catalogados como fracasos. Los proyectos exitosos reportan en promedio cinco años y medio funcionando, lo cual induce a pensar que el periodo de recuperación de seis años para proyectos maderables, recalculado en la evaluación 2004-2008 (CONAFOR 2008), muy probablemente será alcanzado.

En los estados de Durango, Guerrero y Oaxaca se localizaron 85 de los proyectos maderables; los 11 restantes se localizaron en Jalisco, Michoacán y Quintana Roo (figura 1). El número reducido de proyectos en los tres últimos estados, se debe a que los ejidos y comunidades desarrollaron, en su mayoría proyectos no maderables, como el ecoturismo, turismo alternativo, plantas embotelladoras de agua de manantial, extracción de resina, elaboración de artesanías, producción de hongos, entre otros (CONAFOR 2008).

Asimismo, se encontró que el estatus de un proyecto maderable se relacionó con el año en que se otorgó el financiamiento. La prueba ji-cuadrada fue de 10,9 con significancia estadística $(P<0,05)$, lo que indica que existe asociación. Lo anterior se puede explicar por los cambios de directivos y personal operativo del programa entre 2005 y 2006, provocados por el cambio de gobierno federal en México. El porcentaje de fracaso para 2005 fue de casi $47 \%$ y para 2006 fue de poco más de $35 \%$ (figura 2).

Análisis de asociación y correlación. En esta sección se presentan los resultados de la prueba de ji-cuadrada y su significancia estadística (cuadro 2).

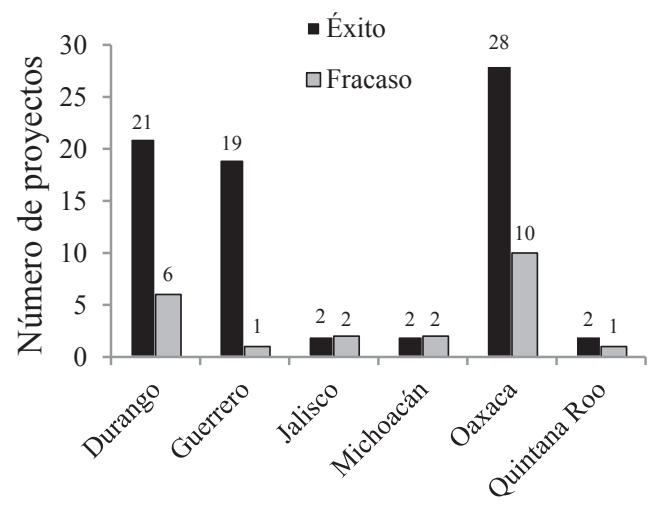

Figura 1. Distribución de proyectos maderables según su estatus por estado.

Distribution of wood projects by status and by state. 


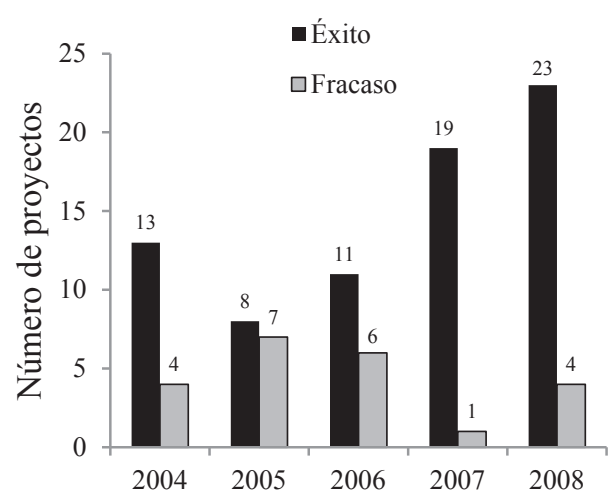

Figura 2. Distribución de proyectos maderables según su estatus en el periodo de 2004 a 2008. 2004 to 2008

Distribution of wood projects by status in the period from

Cuadro 2. Resultados de la prueba ji-cuadrada.

Outcomes of the chi-square test.

\begin{tabular}{llc}
\hline \multicolumn{1}{c}{ Bloque } & \multicolumn{1}{c}{ Factores } & Ji-cuadrada \\
\hline Social & Capital social & $30,1 * * * *$ \\
& Indigenismo & $2,1 \mathrm{~ns}$ \\
& Tipo de propiedad & $0,9 \mathrm{~ns}$ \\
\hline Económico & Finalidad del proyecto & $13,2 * *$ \\
& Tipología del productor & $10,2 * * *$ \\
& Funcionamiento de la empresa & $10,5 * * *$ \\
& Grado de avance del proyecto & $5,6 * *$ \\
\hline Ambiental & Relación de superficie forestal & $6,1 * *$ \\
\hline
\end{tabular}

*: $P<0,10$;**: $P<0,05 ; * * *: P<0,01 ; * * * *: P<0,001 ;$ ns: $P>0,10$.
Con el bloque social, para el contraste del capital social contra el estatus del proyecto, se encontró un valor ji-cuadrada de alta significancia, lo que sugiere que existe una fuerte asociación entre ambos factores. En lo que respecta al contraste de los factores indigenismo y tipo de propiedad, cada uno contra el estatus del proyecto, la prueba jicuadrada no reportó significancia (cuadro 2).

En la exploración de otra medida de asociación entre los niveles de los factores del bloque social y el estatus del proyecto, así como en la identificación de problemas de colinealidad entre los distintos niveles de los factores, la matriz de correlaciones tetracóricas del bloque social proporcionó en la columna de estatus la magnitud y el tipo de influencia (positiva o negativa) con el éxito del proyecto (cuadro 3). Los niveles de los factores del bloque social que tuvieron relación positiva con el éxito de un proyecto maderable fueron: capital social alto, no indígena y ejido. También existió alta correlación entre los niveles de los factores tipo de propiedad e indigenismo (cuadro 3). Por la condición predominante del indigenismo en las zonas forestales de México, fue de interés incluir este factor en el análisis. Por lo tanto, los niveles de los factores del bloque social incluidos en el modelo logístico asociado con la probabilidad de éxito fueron: capital social alto y no indígena. Para el modelo logístico asociado con la probabilidad de fracaso, los niveles de factores relevantes fueron: capital social bajo, capital social medio y sí indígena.

El bloque económico indudablemente fue determinante para el éxito o fracaso de los proyectos maderables. En el cuadro 2 se presentan los valores de la prueba ji-cuadrada de los factores: finalidad del proyecto, tipología del productor, funcionamiento de la empresa, y grado de avance del proyecto, cada uno contra el estatus del proyecto. Los valores encontrados fueron de 13,2 con significancia, 10,2 con alta significancia, 10,5 con alta significancia y de 5,6 con significancia, respectivamente para cada uno de los facto-

Cuadro 3. Matriz de correlaciones tetracóricas del bloque social.

Tetrachoric correlation matrix of the social block.

\begin{tabular}{|c|c|c|c|c|c|c|c|}
\hline \multirow{2}{*}{$\begin{array}{c}\text { Bloque social } \\
\text { Factores }\end{array}$} & \multirow{2}{*}{$\frac{\text { Factores }}{\text { Nivel }}$} & \multirow{2}{*}{$\begin{array}{c}\text { Estatus } \\
\text { Éxito }\end{array}$} & \multicolumn{3}{|c|}{ Capital social } & \multicolumn{2}{|c|}{ Indigenismo } \\
\hline & & & Alto & Medio & Bajo & Sí & No \\
\hline Estatus & Éxito & 1 & - & - & - & - & - \\
\hline \multirow[t]{3}{*}{ Capital social } & Alto & 0,79 & 1 & - & - & - & - \\
\hline & Medio & $-0,42$ & -1 & 1 & - & - & - \\
\hline & Bajo & $-0,73$ & -1 & -1 & 1 & - & - \\
\hline \multirow[t]{2}{*}{ Indigenismo } & Sí indígena & $-0,26$ & 0,26 & $-0,31$ & 0,08 & 1 & - \\
\hline & No indígena & 0,26 & $-0,26$ & 0,31 & $-0,08$ & -1 & 1 \\
\hline \multirow[t]{2}{*}{ Tipo de propiedad } & Comunidad & $-0,17$ & 0,32 & $-0,37$ & 0,08 & 0,99 & $-0,99$ \\
\hline & Ejido & 0,17 & $-0,32$ & 0,37 & $-0,08$ & $-0,99$ & 0,99 \\
\hline
\end{tabular}


res estudiados. Se encontró que $45,45 \%$ de los proyectos para adquisición de aserraderos fracasaron, siendo el principal motivo la inadecuada transferencia de tecnología (en su mayoría extranjera), aunado con la falta de capacitación en la operación y el mantenimiento de la maquinaria, problemas con las refacciones y falta de estudios técnicos ${ }^{1}$.

Los niveles de los factores del bloque económico que tuvieron correlación positiva con el éxito de un proyecto maderable fueron: finalidad del proyecto (abastecimiento, aprovechamiento y equipamiento), tipología del productor (tipo IV), funcionamiento de la empresa (sí empresa), y el grado de avance del proyecto (continuación) (cuadro 4). Puesto que se encontró una correlación alta entre los niveles de los factores finalidad del proyecto y tipología del productor, se utilizó el factor finalidad del proyecto debido a que presentó una distribución homogénea, a diferencia del factor tipología del productor que concentra el $75 \%$ de los proyectos maderables en el nivel tipo IV. Dentro del factor finalidad del proyecto, se excluyó el aprovechamiento debido a que en el modelo de estudio, no contribuye para explicar el éxito o el fracaso de los proyectos.

Los niveles de los factores del bloque económico incluidos en el modelo logístico asociado con la probabi-

Comunicación personal. Ing. Jesús Hernández (informante clave de Oaxaca). lidad de éxito fueron: abastecimiento, equipamiento, sí empresa y proyecto continuación. Para el modelo logístico asociado con la probabilidad de fracaso fueron: adquisición, muebles, estufas de secado, no empresa y proyecto nuevo.

En lo referente al bloque ambiental, el factor considerado fue la relación de superficie forestal respecto de la superficie total. Se consideró una relación forestal alta, si el núcleo agrario posee un recurso forestal de cuando menos la mitad de su territorio; en caso contrario, una relación forestal baja.

Para el contraste de la relación superficie forestal contra estatus del proyecto, el valor ji-cuadrada reportado fue de 6,1 con alta significancia (cuadro 2). La correlación tetracórica fue positiva $(0,42)$ con el éxito cuando la relación de superficie forestal fue alta, y obviamente negativa cuando la relación forestal fue baja.

A continuación, se presentan dos modelos de regresión logística, uno para la probabilidad de éxito y el correspondiente para el fracaso. Lo anterior es pertinente debido a que el complemento de los factores de éxito no necesariamente tiene un efecto negativo para el fracaso.

Modelo asociado a la probabilidad de éxito. Este fue construido considerando todos los niveles de los factores que tuvieron relación positiva con el éxito de un proyecto

Cuadro 4. Matriz de correlaciones tetracóricas del bloque económico.

Tetrachoric correlation matrix of the economic block.

\begin{tabular}{|c|c|c|c|c|c|c|c|c|}
\hline Bloque económico & Factores & Estatus & \multicolumn{6}{|c|}{ Finalidad del proyecto } \\
\hline Factores & Nivel & Éxito & 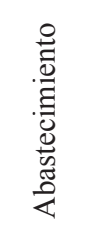 & 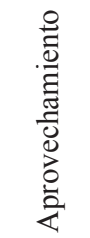 & 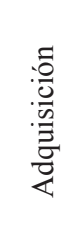 & 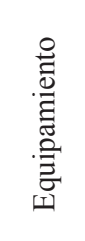 & $\underset{\stackrel{\mathbb{E}}{\rightleftarrows}}{\stackrel{\mathscr{E}}{\Xi}}$ & $\frac{\frac{0}{0}}{\stackrel{0}{0}}$ \\
\hline Estatus & Éxito & 1 & - & - & - & - & - & - \\
\hline \multirow[t]{6}{*}{ Finalidad del proyecto } & Abastecimiento & 0,18 & 1 & - & - & - & - & - \\
\hline & Aprovechamiento & 1 & -1 & 1 & - & - & - & - \\
\hline & Adquisición & $-0,46$ & -1 & -1 & 1 & - & - & - \\
\hline & Equipamiento & 0,37 & -1 & -1 & -1 & 1 & - & - \\
\hline & Estufas & $-0,15$ & -1 & -1 & -1 & -1 & 1 & - \\
\hline & Muebles & $-0,23$ & -1 & -1 & -1 & -1 & -1 & 1 \\
\hline \multirow[t]{3}{*}{ Tipología del productor } & Tipo II & $-0,47$ & 0,52 & -1 & 0,22 & -1 & -1 & -1 \\
\hline & Tipo III & $-0,40$ & $-0,26$ & $-0,07$ & 0,87 & -1 & -1 & -1 \\
\hline & Tipo IV & 0,51 & $-0,03$ & 0,17 & $-0,86$ & 1 & 1 & 1 \\
\hline \multirow[t]{2}{*}{ Funcionamiento de la empresa } & Sí empresa & 0,54 & 0,01 & $-0,11$ & $-0,18$ & $-0,00$ & 0,19 & 0,26 \\
\hline & No empresa & $-0,54$ & $-0,01$ & 0,11 & 0,18 & 0,00 & $-0,19$ & $-0,26$ \\
\hline \multirow[t]{2}{*}{ Avance del proyecto } & Continuación & 0,43 & 0,41 & $-0,18$ & $-0,28$ & $-0,04$ & 0,26 & $-0,28$ \\
\hline & Nuevo & $-0,43$ & $-0,41$ & 0,18 & 0,28 & 0,04 & $-0,26$ & 0,28 \\
\hline
\end{tabular}


maderable. La salida de la regresión logística (cuadro 5) muestra el valor y significancia estadística de los factores.

El capital social alto fue el factor con mayor peso y significancia; en cambio, los factores de abastecimiento, sí empresa y proyecto continuación fueron no significativos (cuadro 5). Tomando en cuenta solo los factores significativos, se procedió a realizar un refinamiento del modelo de éxito (cuadro 6).

De donde, el modelo logístico refinado para el éxito de los proyectos forestales comunitarios fue:

$$
P_{1}=\frac{1}{1+e^{-(-2,38+3,84 * \text { Capital social alto }+1,28 * \text { No indigena }+2,04 * \text { Equipamiento }+1,74 * \text { Relación forestal alta })}}
$$

Cuadro 5. Parámetros estimados del modelo completo de éxito. Estimated parameters of the full model of success.

\begin{tabular}{lccc}
\hline \multicolumn{1}{c}{ Factores } & Coeficientes & $\begin{array}{c}\text { Intervalo de confianza } \\
(90 \%)\end{array}$ \\
\hline Capital social alto & $3,33 * * *$ & 1,62 & 5,03 \\
No indígena & $1,71 * *$ & 0,34 & 3,08 \\
Equipamiento & $1,80 *$ & 0,14 & 3,47 \\
Abastecimiento & $0,40 \mathrm{~ns}$ & $-1,12$ & 1,93 \\
Sí empresa & $0,39 \mathrm{~ns}$ & $-0,94$ & 1,73 \\
Proyecto continuación & $1,17 \mathrm{~ns}$ & $-0,36$ & 2,70 \\
Relación forestal alta & $1,65 * *$ & 0,35 & 2,95 \\
Constante de ajuste & $-2,92 * * *$ & $-4,57$ & $-1,27$ \\
\hline$*: P<0,10 ; * *: P<0,05 ; * * *: P<0,01 ; * * *: P<0,001 ; \mathrm{ns}: P>0,10$.
\end{tabular}

Cuadro 6. Parámetros estimados del modelo refinado de éxito. Estimated parameters of the refined model of success.

\begin{tabular}{lccc}
\hline \multicolumn{1}{c}{ Factores } & Coeficientes & $\begin{array}{c}\text { Intervalo de confianza } \\
(90 \%)\end{array}$ \\
\hline Capital social alto & $3,84 * * * *$ & 2,36 & 5,32 \\
No indígena & $1,28 *$ & 0,13 & 2,43 \\
Equipamiento & $2,04 * *$ & 0,47 & 3,60 \\
Relación forestal alta & $1,74 * *$ & 0,42 & 3,07 \\
Constante de ajuste & $-2,38 * *$ & $-3,90$ & $-0,87$ \\
\hline
\end{tabular}

$*: P<0,10 ; * *: P<0,05 ; * * *: P<0,01 ; * * * *: P<0,001$.

El cuadro 6 muestra la aportación y significancia de los factores que conforman el modelo [1]. Con dichos valores y sin considerar la constante de ajuste se encontró el porcentaje en que contribuye cada factor a la probabilidad de éxito. Los resultados fueron: capital social alto 43,2 \%, condición no indígena $14,4 \%$, finalidad de equipamiento $22,9 \%$ y relación superficie forestal alta 19,5\%.
Las pruebas de bondad de ajuste del modelo refinado de éxito fueron satisfactorias, obteniéndose que el logaritmo de la verosimilitud, únicamente con la constante de ajuste, fuera de $-51,7$. Una vez introducidos los factores y con cinco iteraciones, el logaritmo de la verosimilitud resultante fue de -30,3 que implica una reducción del 41,4\%. La prueba de ji-cuadrada para la bondad de ajuste del modelo fue de 42,8 con una alta significancia $(P<0,001)$. La tabla de contingencia muestra que el modelo clasifica correctamente el 87,5\% de las observaciones y, por último, la prueba de Hosmer y Lemeshow generó un valor ji-cuadrada de 1,91 con ausencia de significancia $(P=0,861)$, lo que indica un buen ajuste del modelo logístico.

Por otro lado, el grupo de informantes clave indicó que los factores que condicionaron el éxito de un proyecto maderable fueron: en Durango, el acompañamiento institucional y el interés de los beneficiarios; en Oaxaca, el capital social y los servicios técnicos forestales; en Michoacán, la transparencia, el asambleísmo, el liderazgo de los representantes, los servicios técnicos forestales y políticas públicas adecuadas; en Jalisco, el funcionamiento de las asambleas, acuerdos para el aprovechamiento forestal, la apropiación de las actividades forestales, la formación de técnicos comunitarios, la rendición de cuentas y la transparencia; y en Guerrero, la consistencia del acompañamiento institucional y de los servicios técnicos forestales, así como el fortalecimiento del capital social de los ejidos y comunidades.

Modelo asociado a la probabilidad de fracaso. Este fue construido considerando todos los niveles de los factores que tuvieron relación negativa con el éxito de un proyecto maderable. Los resultados de la regresión logística (cuadro 7) muestran el valor y significancia de los factores correspondientes.

El capital social bajo fue el factor con mayor peso y significancia, los factores de adquisición, no empresa, proyecto nuevo y relación forestal baja fueron no significativos (cuadro 7). Considerando sólo los coeficientes significativos, se procedió a realizar un refinamiento del modelo de fracaso. Además, se incluyó el factor relación forestal baja, el cual resultó significativo en el modelo refinado, como consecuencia a la reducción de la colinealidad al eliminar los factores no significativos (cuadro 8).

De donde, el modelo logístico refinado para el fracaso de los proyectos forestales comunitarios fue: 


$$
P_{2}=\frac{1}{1+e^{-(6,64-6,50 * \text { Capital social bajo }-4,12 * \text { Capital social medio }-2,23 * \text { Si indigena }-2,22 * \text { Estufas }-3,11 * \text { Muebles }-1,35 * \text { Relación forestal baja })}}
$$

Cuadro 7. Parámetros estimados del modelo completo de fracaso. Estimated parameters of the full model of failure.

\begin{tabular}{lccc}
\hline \multicolumn{1}{c}{ Factores } & Coeficientes & $\begin{array}{c}\text { Intervalo de confianza } \\
(90 \%)\end{array}$ \\
\hline Capital social bajo & $-5,39 * * *$ & $-8,15$ & $-2,63$ \\
Capital social medio & $-3,33 * * *$ & $-5,33$ & $-1,32$ \\
Sí indígena & $-2,07 *$ & $-3,91$ & $-0,23$ \\
Adquisición & $-0,98 \mathrm{~ns}$ & $-2,88$ & 0,93 \\
Estufas de secado & $-2,63 * *$ & $-4,56$ & $-0,70$ \\
Muebles & $-3,25 * * *$ & $-5,28$ & $-1,22$ \\
No empresa & $-0,71 \mathrm{~ns}$ & $-2,27$ & 0,85 \\
Proyecto nuevo & $-1,17 \mathrm{~ns}$ & $-2,80$ & 0,46 \\
Relación forestal baja & $-1,27 \mathrm{~ns}$ & $-2,59$ & 0,05 \\
Constante de ajuste & $7,47 * * * *$ & 4,60 & 10,34 \\
\hline$* 2<0,10 ; *: P<0,05 ; * *: P<0,01 ; * * *$ & $P<0,001 ; n: P>0,10$
\end{tabular}

*: $P<0,10$;**: $P<0,05 ; * * *: P<0,01$; ***: $P<0,001 ;$ ns: $P>0,10$.

Cuadro 8. Parámetros estimados del modelo refinado de fracaso. Estimated parameters of the refined model of failure.

\begin{tabular}{llcc}
\hline \multicolumn{1}{c}{ Factores } & Coeficientes & $\begin{array}{c}\text { Intervalo de confianza } \\
(90 \%)\end{array}$ \\
\hline Capital social bajo & $-6,50 * * * *$ & $-9,03$ & $-3,96$ \\
Capital social medio & $-4,12 * * * *$ & $-5,97$ & $-2,26$ \\
Sí indígena & $-2,23 * * *$ & $-3,54$ & $-0,92$ \\
Estufas de secado & $-2,22 * *$ & $-4,04$ & $-0,40$ \\
Muebles & $-3,11 * * *$ & $-4,99$ & $-1,23$ \\
Relación forestal baja & $-1,35 *$ & $-2,62$ & $-0,09$ \\
Constante de ajuste & $6,64 * * * *$ & 4,20 & 9,08 \\
\hline
\end{tabular}

*: $P<0,10$;**: $P<0,05 ; * * *: P<0,01$; ***: $P<0,001$; ns: $P>0,10$.

El cuadro 8 exhibe la aportación y significancia de los factores que conforman el modelo [2]. Con dichos coeficientes y sin considerar la constante de ajuste se encontró la contribución porcentual de cada uno de los factores a la probabilidad de fracaso. Los resultados fueron: capital social bajo $33,3 \%$, capital social medio $21,1 \%$, sí indígena $11,4 \%$, estufas de secado $11,4 \%$, fábrica de muebles 15,9 $\%$ y relación forestal baja $6,9 \%$.

Las pruebas de bondad de ajuste del modelo refinado de fracaso fueron satisfactorias. El logaritmo de la verosimilitud, sólo con la constante de ajuste, fue de -51,7.
Una vez introducidos los factores y con cinco iteraciones, el logaritmo de la verosimilitud fue de $-26,4$ que implica una reducción de $49 \%$. La prueba de ji-cuadrada para la bondad de ajuste del modelo fue de 50,6 con una alta significancia $(P<0,001)$. La tabla de clasificación muestra que el modelo clasifica correctamente el $87,5 \%$ de las observaciones y, por último, la prueba de Hosmer y Lemeshow generó un valor ji-cuadrada de 1,98 con ausencia de significancia $(P=0,739)$, lo que indica un buen ajuste del modelo logístico.

Por otro lado, las principales causas del fracaso de los proyectos maderables de acuerdo con la información proporcionada por los informantes clave fueron: la falta de acuerdos al interior (21,6\%); el desinterés que tienen las nuevas autoridades o los miembros del ejido o comunidad $(13,5 \%)$; la desorganización interna $(10,8 \%)$; los problemas relacionados con el narcotráfico $(8,1 \%)$; la falta de capacitación técnica, los problemas de litigios, el suministro de refacciones, la tala clandestina, y los grupos internos (5,4 \% cada uno); la corrupción, la mala asesoría técnica, los contratos de luz, la falta de rentabilidad, los problemas con el suministro de materias primas, los problemas de integración en el aprovechamiento forestal y los partidos políticos (2,7\% cada uno).

\section{DISCUSIÓN}

Encontrar que el capital social con un nivel alto es el factor de mayor relevancia para el éxito de los proyectos forestales comunitarios está en correspondencia con trabajos previos, ya que una comunidad con esta característica ha cultivado los elementos necesarios para institucionalizar sus procesos y generar mecanismos que fomenten el éxito de sus actividades. Esto indica que la presencia de acuerdos, reglamentos y el respeto entre personas que comparten diversas actividades cuyos intereses individuales podrían no coincidir, propician una mejor colaboración y cooperación para hacer que un proyecto comunitario sea exitoso. Resulta consecuente que un capital social bajo o medio sea un factor de fracaso, en complementariedad de que el capital social alto fomenta el éxito (Bray y Merino 2004, Rubio et al. 2007, Madrid et al. 2009).

El factor que considera la presencia de población indígena en los núcleos agrarios tuvo una influencia moderada en la probabilidad de fracaso. Este resultado muestra que los esfuerzos de política pública realizados en los ejidos y comunidades indígenas no han sido suficientes ni adecuados. Por lo que la administración pública debe mejorar la interacción con las comunidades indígenas, en el sentido de promover la capacitación de sus miembros en los objetivos de los proyectos, o en su caso, promover el desa- 
rrollo de proyectos especiales que incorporen elementos culturales propios y así las comunidades creen, mantengan y mejoren sus empresas forestales, sin menoscabo de sus usos y costumbres (Bray y Merino 2004).

En lo que se refiere al tipo de propiedad social (es decir, si es ejido o comunidad), se encontró que el éxito o fracaso de un proyecto maderable no se encuentra asociado con el tipo de propiedad social donde se realiza. Este resultado sugiere que aunque el régimen de tenencia agrario es diferente, la organización al interior de estos suele ser semejante, de ahí que su incidencia sobre el éxito o fracaso de los proyectos no sea significativa. Este resultado concuerda con estudios previos donde se encontró que no existe relación entre el tipo de propiedad con el fracaso en el uso de los recursos naturales (Madrid et al. 2009).

Respecto a la finalidad del proyecto, los resultados indican que la complejidad del proyecto representa una medida de riesgo que debe cuidarse si se desea pasar de un aprovechamiento primario a una industria de transformación, como la fabricación de muebles o el estufado de la madera (Bray et al. 2007). Futuras investigaciones pueden abordar la medición de lo que la complejidad del proyecto agrega a la probabilidad de fracaso así como las actividades complementarias que deberían observarse para evitarlo.

El funcionamiento de la empresa forestal comunitaria no modifica la probabilidad de éxito ni la de fracaso. Este resultado indica que en los ejidos y comunidades forestales, esta estructura organizativa aún no se encuentra consolidada, por lo que su adopción y operación ha sido limitada. En el informe de la Comisión Nacional Forestal, se reportó que debido a las dificultades de los ejidos y comunidades para tomar acuerdos, sólo $50 \%$ de las empresas constituidas se encontraba operando (CONAFOR 2003).

Se encontró que ni la probabilidad de éxito ni la de fracaso, fueron condicionadas por el grado de avance del proyecto (nuevo o ampliación). Lo anterior tiene sentido ya que para emprender proyectos con mayor grado de avance, son requeridas mayores inversiones, mercados especializados, mayor disponibilidad de materia prima, transferencia de nueva tecnología y procesos administrativos más complejos. Es decir, un proyecto nuevo es proclive al fracaso por lo que se desconoce de él, mientras que un proyecto de continuación (ampliación) confronta nuevos retos que lo pueden llevar al fracaso. Este resultado exhibe que otros factores tienen mayor prioridad que el grado de avance del proyecto.

Por último, los resultados exhiben la contribución del factor relación superficie forestal alta al éxito, lo que concuerda con el hecho de que los ejidos y comunidades forestales cuyo territorio, en su mayor parte, está cubierto por bosques y selvas, pueden sufragar costos que no se tenían previstos en el proyecto técnico, en comparación con aquellos que únicamente cuentan con los recursos asignados.

No obstante que las experiencias de éxito no son fácilmente reproducibles, debido a la multiplicidad de factores interactuantes, es importante tomar en cuenta los factores relevantes en los casos de éxito, cuando se pone en marcha proyectos con enfoque comunitario en América Latina, como los proyectos de REDD+ (Alcorn 2014). Entre las lecciones de la silvicultura comunitaria que pueden extenderse para facilitar el éxito de los proyecto de REDD+, se pueden considerar los factores que: favorecen el funcionamiento de los proyectos u obstaculizan las posibilidades de éxito de los mismos; impulsan la conservación y la buena gestión, y las intervenciones externas (Alcorn 2014).

Una de las ventajas de los modelos presentados en este trabajo es que integran los factores ambientales, económicos y sociales de tipo cualitativo, en contraste con trabajos previos (Merino 2003, Bray et al. 2007, Madrid et al. 2009, López et al. 2010).

\section{CONCLUSIONES Y RECOMENDACIONES}

El conocimiento de los factores que influyen en el desarrollo de los proyectos forestales comunitarios, representa la diferencia entre el éxito o fracaso de los mismos, de ahí su relevancia. Las experiencias de la silvicultura comunitaria en el caso mexicano son susceptibles de tomarse en cuenta en otros países de América Latina, incluso para facilitar el éxito de los proyectos de REDD+, dadas las condiciones semejantes entre nuestros países y del manejo de bosques en las comunidades rurales que deviene de los tipos de bosques que compartimos. En este trabajo se encontró que el capital social es el factor más importante para explicar el éxito o fracaso de los proyectos forestales comunitarios, el nivel alto para la probabilidad de éxito y los niveles medio y bajo para explicar la probabilidad de fracaso, explicación que oscila alrededor del $50 \%$ en ambos casos. Otros factores importantes que mejoran las condiciones para el éxito de los proyectos forestales es el porcentaje de superficie forestal en la comunidad y el objetivo final del proyecto. El hecho de que los proyectos mejoraron su probabilidad de éxito en las comunidades no indígenas sugiere la necesidad de un mayor apoyo para capacitación en las comunidades indígenas acerca de los proyectos productivos.

Derivado de los resultados obtenidos, se hacen tres recomendaciones para atender los factores que condicionan la probabilidad de éxito o fracaso en los proyectos forestales comunitarios:

1. Los programas de política pública forestal que atienden la demanda de proyectos forestales comunitarios, deben impulsar acciones para: a) fortalecer la planeación y organización comunitaria, b) promover el intercambio de experiencias comunitarias, c) realizar foros regionales entre comunidades y d) atender la formación de técnicos comunitarios.

2. En los ejidos y comunidades forestales indígenas se debe promover un esquema particular de fortalecimiento del capital social que integre los reglamentos internos y el apego a ellos, con base en sus usos y costumbres. 
3. Realizar un seguimiento especial de los proyectos comunitarios en los ejercicios fiscales donde ocurren cambios en la administración pública a nivel nacional, con el objetivo de identificar posibles influencias negativas o positivas asociadas.

\section{AGRADECIMIENTOS}

Los autores agradecen la información proporcionada por el grupo de informantes clave: en Durango a José Galván y José de Jesús Valdez; en Guerrero a Eduardo Giadans y Teodoro Morales; en Jalisco a Víctor Hugo Martínez y Ana Bertha Santoyo; en Michoacán a Adolfo Chávez y Raúl Silva; en Oaxaca a Jesús Hernández, y en Quintana Roo a Blas Santiago, Bernabé del Ángel y Raúl Pérez.

\section{REFERENCIAS}

Alcorn JB. 2014. Lecciones aprendidas de la silvicultura comunitaria en América Latina y su relevancia para REDD+. Programa de Carbono Forestal, Mercados y Comunidades (FCMC) auspiciado por USAID. Washington, EE.UU. Agencia de los Estados Unidos para el Desarrollo Internacional. 72 p.

Agrawal A. 1995. Population pressure = forest degradation: an oversimplistic equation? Unasylva 46 (181): s.p.

Bray DB, L Merino, P Negreros, G Segura, JM Torres y H Vester. 2003. Mexico's community-managed forests as a global model for sustainable landscapes. Conservation Biology 17(3): 672-677.

Bray DB, L Merino. 2004. La experiencia de las comunidades forestales en México; Veinticinco años de silvicultura y construcción de empresas forestales comunitarias. Distrito Federal, México. Secretaría de Medio Ambiente y Recursos Naturales, Instituto Nacional de Ecología, Consejo Civil Mexicano para la Silvicultura Sostenible, Fundación Ford. 272 p.

Bray DB, E Durán, L Merino, JM Torres, A Velázquez. 2007. Nueva evidencia: los bosques comunitarios de México protegen el ambiente, disminuyen la pobreza y promueven paz social. Distrito Federal, México. Universidad Nacional Autónoma de México, Centro de Investigación y Docencia Económica, CIIDIR-OAXACA, Instituto Politécnico Nacional, Universidad Internacional de Florida. 23 p.

CONAFOR (Comisión Nacional Forestal, MX). 2003. Gestión comunitaria para el uso sustentable de los bosques. México DF, México. CONAFOR. 120 p. (Informe final PROCy-
MAF).

CONAFOR (Comisión Nacional Forestal, MX). 2008. Programa De Desarrollo Forestal Comunitario PROCYMAF II. México DF, México. CONAFOR. 137 p. (Informe final de la evaluación externa 2004-2008).

CONAFOR (Comisión Nacional Forestal, MX). 2012. Silvicultura (Silvicultura Comunitaria). Consultado 26 nov. 2012. Disponible en http://www.conafor.gob.mx/portal/index. $\mathrm{php} /$ temas-forestales/silvicultura

FAO (Food and Agriculture Organization of the United Nations, IT). 1978. Forestry for local community development. Forestry Paper 7. Rome, Italy. FAO. 117 p.

Gerez P, SE Purata. 2008. Guía Práctica Forestal de Silvicultura Comunitaria. México DF, México. SEMARNAT/ CONAFOR/CCMSS. 73 p.

INEGI (Instituto Nacional de Estadística y Geografía, MX). 2008. Censo Agropecuario 2007 (VIII Censo Agrícola, Ganadero y Forestal). Consultado 26 nov. 2012. Disponible en http://www.inegi.org.mx/est/contenidos/proyectos/agro/ ca2007/resultados_agricola/default.aspx

Ledesma RD, G Macbeth, P Valero-Mora. 2011. Software for computing the tetrachoric correlation coefficient. Revista Latinoamericana de Psicología 43: 181-189.

Leslie A. 1987. Foreword. In Westoby J ed. The Purpose of Forest: The Follies of Development. Oxford, UK. Blackwell. p. vii-xiii.

López F, A Velázquez, L Merino. 2010. Explorando los determinantes del buen manejo forestal comunitario. Interciencia 35(8): 560-567.

Luján A. 2003. Forestería comunitaria: una acción de base para el desarrollo sustentable en México. Relaciones 24(94): $267-$ 283.

Madrid L, JM Nuñez, G Quiroz, Y Rodríguez. 2009. La propiedad social forestal en México. Investigación ambiental 1(2): 179-196.

Merino L. 2003. Los bosques de México, reflexiones en torno a su manejo y conservación. Ciencias 72: 59-67.

Ostrom E, K Ahn. 2003. Una perspectiva del capital social desde las ciencias sociales: capital social y acción colectiva. Revista Mexicana de Sociología 1: 155-233.

Pokorny B, C Sabogal, W de Jon, D Stoian, B Louman, P Pacheco, N Porro. 2009. Experiencias y retos del manejo forestal comunitario en América Tropical. Recursos Naturales y Ambiente 54: 81-89.

Rubio M, OP Herrera, ME Pérez. 2007. El capital social en comunidades del Distrito de Ixtlán: estudios de casos de la Trinidad, Santa Catarina Ixtepeji e Ixtlán de Juárez. Naturaleza y Desarrollo 5(1): 17-26. 
Apéndice 1. Proyectos forestales maderables comunitarios con financiamiento gubernamental en México.

Community forest timber-yielding projects under government funding in Mexico.

\begin{tabular}{|c|c|c|c|c|c|}
\hline Estado & Núcleo agrario & Tenencia & Financiamiento & Clima-Hábitat & Número de proyectos \\
\hline \multirow[t]{19}{*}{ Durango } & Borbollones & Ejido & Gobierno Federal & Templado-Bosque & 1 \\
\hline & Ciénega de La Vaca & Ejido & Gobierno Federal & Templado-Bosque & 1 \\
\hline & Duraznitos y Picacho & Ejido & Gobierno Federal & Templado-Bosque & 1 \\
\hline & El Maguey & Ejido & Gobierno Federal & Templado-Bosque & 1 \\
\hline & El Salto y Anexos & Ejido & Gobierno Federal & Templado-Bosque & 1 \\
\hline & El Tarahumar y Bajío & Comunidad & Gobierno Federal & Templado-Bosque & 1 \\
\hline & El Zorrillo y Anexos & Ejido & Gobierno Federal & Templado-Bosque & 1 \\
\hline & La Ciudad & Ejido & Gobierno Federal & Templado-Bosque & 1 \\
\hline & La Florida & Ejido & Gobierno Federal & Templado-Bosque & 1 \\
\hline & La Manga y Anexos & Ejido & Gobierno Federal & Templado-Bosque & 1 \\
\hline & Llano Grande & Ejido & Gobierno Federal & Templado-Bosque & 1 \\
\hline & Salto de Camellones & Ejido & Gobierno Federal & Templado-Bosque & 2 \\
\hline & San Bartolo & Ejido & Gobierno Federal & Templado-Bosque & 1 \\
\hline & San Diego de Tezains & Ejido & Gobierno Federal & Templado-Bosque & 2 \\
\hline & San Pablo & Ejido & Gobierno Federal & Templado-Bosque & 3 \\
\hline & Topia & Ejido & Gobierno Federal & Templado-Bosque & 1 \\
\hline & Vencedores & Ejido & Gobierno Federal & Templado-Bosque & 3 \\
\hline & Las Hacienditas y Anexos & Ejido & Gobierno Federal & Templado-Bosque & 1 \\
\hline & San Bernardino de Milpillas Chico & Comunidad & Gobierno Federal & Templado-Bosque & 3 \\
\hline \multirow[t]{13}{*}{ Guerrero } & Carrizal de Bravo & Ejido & Gobierno Federal & Templado-Bosque & 1 \\
\hline & Cordón Grande & Ejido & Gobierno Federal & Templado-Bosque & 1 \\
\hline & Corrales & Ejido & Gobierno Federal & Templado-Bosque & 1 \\
\hline & El Balcón & Ejido & Gobierno Federal & Templado-Bosque & 3 \\
\hline & El Carrizal & Ejido & Gobierno Federal & Templado-Bosque & 1 \\
\hline & El Durazno & Ejido & Gobierno Federal & Templado-Bosque & 1 \\
\hline & La Concordía & Comunidad & Gobierno Federal & Templado-Bosque & 2 \\
\hline & Los Bajitos & Ejido & Gobierno Federal & Templado-Bosque & 3 \\
\hline & Pascala del Oro & Comunidad & Gobierno Federal & Templado-Bosque & 1 \\
\hline & Platanillo & Ejido & Gobierno Federal & Templado-Bosque & 1 \\
\hline & San Antonio Texas & Ejido & Gobierno Federal & Templado-Bosque & 2 \\
\hline & San Cristóbal & Ejido & Gobierno Federal & Templado-Bosque & 1 \\
\hline & Santa Bárbara & Ejido & Gobierno Federal & Templado-Bosque & 2 \\
\hline \multirow[t]{3}{*}{ Jalisco } & San Juan de Los Potreros & Comunidad & Gobierno Federal & Templado-Bosque & 1 \\
\hline & Concepción de Buenos Aires & Ejido & Gobierno Federal & Templado-Bosque & 1 \\
\hline & Barranca del Calabozo & Ejido & Gobierno Federal & Templado-Bosque & 2 \\
\hline \multirow[t]{4}{*}{ Michoacán } & Barranca Seca & Ejido & Gobierno Estatal & Templado-Bosque & 1 \\
\hline & Cheran & Comunidad & Gobierno Estatal & Templado-Bosque & 1 \\
\hline & Pucuato & Ejido & Gobierno Federal & Templado-Bosque & 1 \\
\hline & Barrio de San Francisco & Comunidad & Gobierno Estatal & Templado-Bosque & 1 \\
\hline
\end{tabular}


Continuación Apéndice 1

\begin{tabular}{|c|c|c|c|c|c|}
\hline \multirow[t]{26}{*}{ Oaxaca } & Ixtlán de Juárez & Comunidad & Gobierno Federal & Templado-Bosque & 2 \\
\hline & La Trinidad & Comunidad & Gobierno Federal & Templado-Bosque & 2 \\
\hline & Nuevo Zoquiapan & Comunidad & Gobierno Federal & Templado-Bosque & 1 \\
\hline & San Andrés Cabecera & Comunidad & Gobierno Federal & Templado-Bosque & 1 \\
\hline & San Andrés El Alto & Comunidad & Gobierno Federal & Templado-Bosque & 2 \\
\hline & San Andrés Nuxiño & Comunidad & Gobierno Federal & Templado-Bosque & 1 \\
\hline & San Antonino El Alto & Comunidad & Gobierno Federal & Tropical-Selva & 2 \\
\hline & San Juan Quiahije & Comunidad & Gobierno Federal & Templado-Bosque & 1 \\
\hline & San Miguel Mixtepec & Comunidad & Gobierno Federal & Templado-Bosque & 3 \\
\hline & San Pedro El Alto & Comunidad & Gobierno Federal & Templado-Bosque & 1 \\
\hline & San Pedro Leapi & Comunidad & Gobierno Federal & Templado-Bosque & 1 \\
\hline & San Sebastián Jilote & Comunidad & Gobierno Federal & Templado-Bosque & 1 \\
\hline & San Vicente Lachixio & Comunidad & Gobierno Federal & Templado-Bosque & 1 \\
\hline & Santa Cruz Itundujia & Comunidad & Gobierno Federal & Templado-Bosque & 1 \\
\hline & Santiago Comaltepec & Comunidad & Gobierno Federal & Templado-Bosque & 1 \\
\hline & Santiago Laxopa & Comunidad & Gobierno Federal & Templado-Bosque & 4 \\
\hline & Santiago Textitlán & Comunidad & Gobierno Federal & Templado-Bosque & 3 \\
\hline & Santiago Xiacuí & Comunidad & Gobierno Federal & Templado-Bosque & 2 \\
\hline & Santo Domingo Coatlán & Comunidad & Gobierno Federal & Templado-Bosque & 1 \\
\hline & San Francisco La Reforma & Comunidad & Gobierno Federal & Templado-Bosque & 1 \\
\hline & San Martin Buenavista & Comunidad & Gobierno Federal & Templado-Bosque & 1 \\
\hline & Santa Catarina Ixtepeji & Comunidad & Gobierno Federal & Templado-Bosque & 1 \\
\hline & Santa Cruz Zenzontepec & Comunidad & Gobierno Federal & Templado-Bosque & 1 \\
\hline & San Sebastian Rio Dulce & Comunidad & Gobierno Federal & Templado-Bosque & 1 \\
\hline & San Pedro Tepalcatepec & Comunidad & Gobierno Federal & Templado-Bosque & 1 \\
\hline & San Esteban Atatlahuca & Comunidad & Gobierno Federal & Templado-Bosque & 1 \\
\hline \multirow[t]{4}{*}{ Quintana Roo } & Francisco Botes & Ejido & Gobierno Federal & Tropical-Selva & 1 \\
\hline & Noh-Bec & Ejido & Gobierno Federal & Tropical-Selva & 1 \\
\hline & Tres Garantías & Ejido & Gobierno Federal & Tropical-Selva & 1 \\
\hline & & & & & Total \\
\hline
\end{tabular}

